\title{
Optimal Tap Configuration for Step-Voltage Regulators Applied to Residential Feeders
}

\author{
Víctor García,Cristina González-Morán, Pablo Arboleya \\ Department of Electrical Engineering \\ University of Oviedo \\ Gijón, Spain
}

\begin{abstract}
In the present paper, a centralized control method of the tap configuration for step-voltage regulators (SVRs) is proposed. First, the problem will be formulated: The power flow equations and the objective function with its constraints will be described for the studied system. Two different objective functions are defined for comparison purposes: On the one hand, the objective will be minimizing the deviations of voltages with respect to their rated values. On the other hand, the minimized voltage scenario is considered. These two cases are compared also to the case base in which there is no regulation. Aspects such as power injected to the grid, power losses and degree of unbalance are analyzed. Focusing on residential feeders, a complete 24-hour span will let us simulate several different load conditions. Moreover, by utilizing a general load model, instead of the constant power model (PQ), and by applying an accurate load demand prediction, more realistic results are achieved. The case studies are implemented in a modified version of the IEEE 123 Node Test Feeder, which includes additional and rearranged regulators.
\end{abstract}

Index Terms-Composite Load Models, Distribution Grids, Optimal Power Flow, Step Voltage Regulators.

\section{INTRODUCTION}

Step Voltage Regulators (SVRs) have been commonly installed in distribution grids to improve voltage profiles. In contrast to other regulation devices, such as shunt capacitors or static var compensators, whose operating principles are based on reactive power injection or consumption, SVRs allow direct voltage variations by modifying the position of their winding taps. Two configurations are available depending on the voltage regulation needs; step-up or to step-down [1].

Up to recent years, the lack of distribution data collected from the utilities, forced the SRV controlled systems to rely only on local measurements, and thus not being able to optimally operate together as a single control entity. With the new generation of monitoring devices (e.g. smart meters and synchrophasors) the amount of information that can be obtained has exponentially increased, allowing the development of studies and novel control techniques for enhanced overall performance of distribution feeders.

Several authors have proposed diverse methods and algorithms to optimize grid design and operation through the tap

Corresponding author email: gonzalezmorcristina@uniovi.es

This work was partially supported by the Spanish Ministry of Science and Innovation under Grant ENE2013-44245-R (MICROHOLO Development of a Holistic and Systematical Approach to AC Microgrids Design and Management) configuration control of the SVRs. For instance, [2] seeks the minimization of the total system power losses through a semi-definite programming (SDP) algorithm. [3] proposes a two-step algorithm able to calculate the optimal placement of SVRs. [4] implements a sorting genetic algorithm to approach the optimal location and the regulator lifetime under variable weather conditions, which raise the uncertainty of the voltage profiles. In the case of [5], the authors analyze in depth the interactions between step-voltage regulators and large photovoltaic systems, regarding voltage quality, unbalance and tap operation times.

In this work, an algorithm to compute the optimal tap positions in SVRs for improving voltage profiles has been defined. The main contribution is that the algorithm is very easy to implement, general for every type of SVR configuration adapted to include residential distribution feeders in distributed and unbalanced power systems. The method will be applied with different consumptions patters to prove that the considered model of load affects the obtained results in a considerable manner; For bulk power flow analysis, the classic constant PQ load model is sufficiently accurate, as the error committed is minuscule, but when trying to take a more precise approach as in distributed systems, load modeling can be a crucial factor. Lighting and heating systems do not follow the constant power behavior, as they are voltage dependent. Even though most of dwelling appliances do behave as constant PQ, given the power electronics involved, the results might differ when the general load model is implemented.

Besides SVRs, other regulation devices might be introduced in this model by following the procedure described in [6].

The structure of the paper is the following: Section II covers the problem formulation, Section III includes the optimization algorithm and the research methodology established by the authors. Section III displays the results obtained. Finally, Section IV concludes the paper.

\section{PROBLEM FORMULATION}

The formulation will allow for the inclusion of distribution feeders, different kinds of loads and SVRs. The whole problem is formulated in matrix form; First, a Step-Votage-Regulator mathematical model will be stated and then the grid and loads will be introduced.

For the SVR modeling, a delta connection has been chose to be described in this section: Open-Delta with Type A regu- 


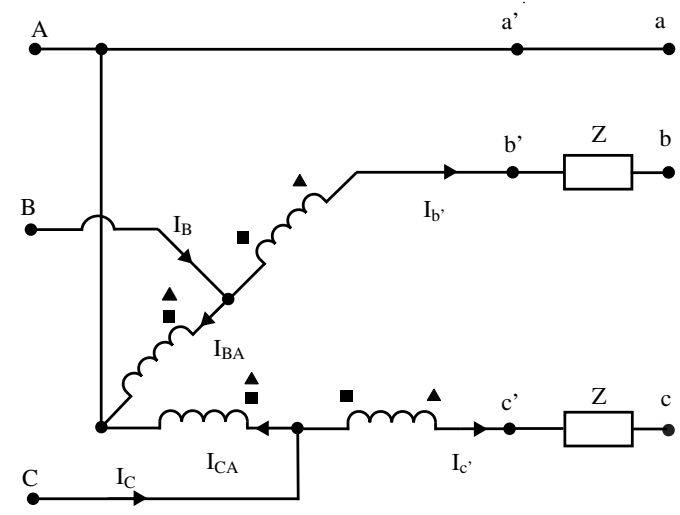

Fig. 1: Open Delta configuration. Type A regulators. $\Delta$ Raise position. $\square$ Lower position.

lators, but any other configuration can be deducted employing the same procedure.

Two single phase regulators can be connected giving rise to a three phase configuration. This is known as an OpenDelta connection. Because there are two regulators connected to three phases, there might be three different configurations. The case in which the two regulators are connected to phases $\mathrm{b}$ and $\mathrm{c}$ is depicted in Fig. 1; The regulators are of type A [1] and are supposed to be in raise position. Two equations are defined to introduce a SVR into the power flow problem: The relationship between voltages and currents at primary and secondary sides respectively.

For the voltage case, it has to be noticed that phases $A$ in the primary and $a$ in the secondary are directly connected, so it can written $A=a^{\prime}=a$. From the figure, the voltages through the first regulator are related as follows:

$$
V_{a^{\prime} b^{\prime}}=V_{A b^{\prime}}=V_{A B}+V_{B b^{\prime}}
$$

Being $N_{1}$ the number of turns for the shunt winding and $N_{2}$ the number of turns for the series winding, the voltages $V_{A B}$ and $V_{B b^{\prime}}$ can be related:

$$
\frac{V_{B b^{\prime}}}{V_{A B}}=\frac{N_{2}}{N_{1}}
$$

Merging (1) and (2) a single equation can be obtained:

$$
V_{a^{\prime} b^{\prime}}=V_{A B}+V_{A B} \frac{N_{2}}{N_{1}}=V_{A B}\left(1+\frac{N_{2}}{N_{1}}\right)
$$

If a the relation $a_{R_{a b}}=1+\frac{N_{2}}{N_{1}}$ is defined then primary and secondary voltages can be directly related as:

$$
V_{a^{\prime} b^{\prime}}=a_{R_{a b}} V_{A B}
$$

The same procedure applies to $V_{c^{\prime} a^{\prime}}$ voltage to obtain:

$$
V_{c^{\prime} a^{\prime}}=a_{R_{c a}} V_{C A}
$$

The matrix equation, for the three primary voltages as functions of the three secondary voltages, is built from, and taking into account that the equation $V_{A B}+V_{B C}+V_{C A}=0$ has to be satisfied:

$$
\left[\begin{array}{l}
V_{A B} \\
V_{B C} \\
V_{C A}
\end{array}\right]=\left(\begin{array}{ccc}
\frac{1}{a_{R_{a b}}} & 0 & 0 \\
-\frac{1}{a_{R_{a b}}} & 0 & -\frac{1}{a_{R_{c a}}} \\
0 & 0 & \frac{1}{a_{R_{c a}}}
\end{array}\right)\left[\begin{array}{l}
V_{a^{\prime} b^{\prime}} \\
V_{b^{\prime} c^{\prime}} \\
V_{c^{\prime} a^{\prime}}
\end{array}\right]
$$

Let us label the matrix in equation (6) as $\mathbf{A}_{R_{v}}$ so the equation is written in compact form:

$$
[\mathbf{V}]_{a b c}^{\mathrm{P}}=\mathbf{A}_{R_{v}}[\mathbf{V}]_{a b c}^{\mathrm{S}^{\mathrm{S}}}
$$

For the studied connection the voltage drops across the regulator impedances are given in matrix form by equation (8).

$$
\left[\begin{array}{l}
V_{a^{\prime} a} \\
V_{b^{\prime} a} \\
V_{c^{\prime} c}
\end{array}\right]=Z\left(\begin{array}{lll}
0 & 0 & 0 \\
0 & 1 & 0 \\
0 & 0 & 1
\end{array}\right)\left[\begin{array}{l}
I_{a} \\
I_{b} \\
I_{c}
\end{array}\right]
$$

There is no voltage drop due to current $I_{a}$ because, as it is deducted from Fig. 1, there is no impedance in phase $a$ because $A=a=a^{\prime}$. Merging equations (6) and (8) we need to relate primary and secondary voltages, avoiding points $a^{\prime}, b^{\prime}$ and $c^{\prime}$. For that propose, first the matrix equation that relates voltages at $a^{\prime}, b^{\prime}$ and $c^{\prime}$ to $a^{\prime}, b^{\prime}$ and $c^{\prime}$ and currents is needed:

$$
\left[\begin{array}{l}
V_{a^{\prime} b^{\prime}} \\
V_{b^{\prime} c^{\prime}} \\
V_{c^{\prime} a^{\prime}}
\end{array}\right]=\left[\begin{array}{l}
V_{a b} \\
V_{b c} \\
V_{c a}
\end{array}\right]+\left(\begin{array}{ccc}
1 & -1 & 0 \\
0 & 1 & -1 \\
-1 & 0 & 1
\end{array}\right)\left[\begin{array}{l}
V_{a^{\prime} a} \\
V_{b^{\prime} b} \\
V_{c^{\prime} c}
\end{array}\right]
$$

The matrix of equation (9) will be labeled as $\mathbf{T}_{\mathrm{DY}}$. It is a singular matrix. Then, replacing (8) into (9) it can be written:

$$
\left[\begin{array}{l}
V_{a^{\prime} b^{\prime}} \\
V_{b^{\prime} c^{\prime}} \\
V_{c^{\prime} a^{\prime}}
\end{array}\right]=\left[\begin{array}{l}
V_{a b} \\
V_{b c} \\
V_{c a}
\end{array}\right]+\mathbf{T}_{\mathrm{DY}} Z\left(\begin{array}{lll}
0 & 0 & 0 \\
0 & 1 & 0 \\
0 & 0 & 1
\end{array}\right)\left[\begin{array}{l}
I_{a} \\
I_{b} \\
I_{c}
\end{array}\right]
$$

Finally, substituting (10) into (6) and writing the resulting expression in compact form, it results (11), which is the expression that summarizes primary and secondary voltages as a function of currents.

$$
[\mathbf{V}]_{a b c}^{\mathrm{P}}=\mathbf{A}_{R_{v}}[\mathbf{V}]_{a b c}^{\mathrm{S}}+Z \mathbf{A}_{R_{v}} \mathbf{T}_{\mathrm{DY} a}[\mathbf{I}]_{a b c}^{\mathrm{S}}
$$

where

$$
\mathbf{T}_{\mathrm{DY} a}=\left(\begin{array}{rrr}
0 & -1 & 0 \\
0 & 1 & -1 \\
0 & 0 & 1
\end{array}\right)
$$

To derive the relationship between primary and secondary currents from Fig. 1 it can be said:

$$
\begin{aligned}
& I_{B}=I_{b}+I_{B A} \\
& I_{C}=I_{c}+I_{C A}
\end{aligned}
$$

Using the number of turns in primary and secondary windings and considering once again the raise position for the regulators 
equations (15) and (16) apply:

$$
\begin{aligned}
\frac{I_{B A}}{I_{b}} & =\frac{N_{2}}{N_{1}} \\
\frac{I_{C A}}{I_{c}} & =\frac{N_{2}}{N_{1}}
\end{aligned}
$$

Merging the last four equations, and writing them in terms of turn relations, it is obtained that:

$$
\begin{aligned}
& I_{B}=I_{b}\left(1+\frac{N_{2}}{N_{1}}\right)=a_{R_{a b}} I_{b} \\
& I_{C}=I_{c}\left(1+\frac{N_{2}}{N_{1}}\right)=a_{R_{c a}} I_{c}
\end{aligned}
$$

Merging (17) and (18) and taking into account that for a three phase three wire connection the equation $I_{A}+I_{B}+I_{C}=0$ must be satisfied, a the matrix equation relating primary and secondary currents is directly deducted:

$$
\left[\begin{array}{c}
I_{A} \\
I_{B} \\
I_{C}
\end{array}\right]=\left(\begin{array}{ccc}
0-a_{R_{a b}} & -a_{R_{c a}} \\
0 & a_{R_{a b}} & 0 \\
0 & 0 & a_{R_{c a}}
\end{array}\right)\left[\begin{array}{c}
I_{a} \\
I_{b} \\
I_{c}
\end{array}\right]
$$

The matrix in equation (19) will be labeled as $\mathbf{A}_{R_{I}}$. (11) and (19) summarize the SVR model. The used algorithm is an unbalanced BFS solver [1] in which linear equations were defined in matrix form including all system KVL and KCL equations:

$$
\mathbf{M} \mathbf{z}^{T}=0
$$

The vector $\mathbf{z}$ contains all complex, three phase system voltages and currents as follows:

$$
\mathbf{z}=\left[\begin{array}{llll}
\mathbf{I}_{B} & \mathbf{I}_{\mathbf{L o a d}} & \mathbf{I}_{\mathbf{G}} & \mathbf{V}
\end{array}\right]
$$

where $\mathbf{I}_{B}, \mathbf{I}_{\text {load }}$ and $\mathbf{I}_{G}$ are vectors including all branch, load and generator currents and $\mathbf{V}$ is a vector including all node voltages in the studied network. The structure of matrix $\mathbf{M}$ is shown in (22).

$$
\mathbf{M}=\left(\begin{array}{c|c|c|c}
\mathbf{Z}_{\alpha \beta 0} & 0 & 0 & -\boldsymbol{\Gamma} \\
\hline \boldsymbol{\Gamma}^{T} & \mathbf{I}_{d} & -\mathbf{I}_{d} & 0
\end{array}\right)
$$

where the matrices $\boldsymbol{\Gamma}$ and $\boldsymbol{\Gamma}^{T}$ are the modified node incidence matrices in which the SVR matrices $\mathbf{A}_{R_{v}}, \mathbf{A}_{R_{I}}$ and $\mathbf{T}_{\mathrm{DY}_{a}}$ are properly included at the corresponding positions where a SVR is connected. A similar procedure is described for conventional transformers (without taps) in [7]. Finally, loads will add the following non liner equations:

$$
\begin{aligned}
\mathbf{P}_{a b c} & =\operatorname{real}\left(\mathbf{A} \mathbf{V}_{\alpha \beta 0} \circ \operatorname{conj}\left[\mathbf{A} \mathbf{I}_{L_{\alpha \beta 0}}\right]\right) \\
\mathbf{Q}_{a b c} & =\operatorname{imag}\left(\mathbf{A} \mathbf{V}_{\alpha \beta 0} \circ \operatorname{conj}\left[\mathbf{A} \mathbf{I}_{L_{\alpha \beta 0}}\right]\right)
\end{aligned}
$$

where the operator $\circ$ is the Hadamard (element-wise) product. This expression is applicable to constant power, current or impedance loads.

\section{OPTIMIZATION ALGORITHM}

The problem can be set out as a nonlinear constrained mixed-integer optimization, as the positions of the taps, which are the control variables, take discrete values. Given that the regulators may remain unchanged for periods of time as high as hours, the run time is not a mandatory feature, so the problem can be approached as a nonlinear constrained continuous optimization. In this way, the programming is simplified without greatly compromising performance, only having to round up the tap positions at the end of the process. The lower admissible voltage limit will be the most critical, so rounding up guarantees it is not surpassed.

The active-set algorithm was chosen; It falls into the category of sequential quadratic programming (SQP), an iterative process based on the approximation of the Hessian of the Lagrangian function, by means of a quasi-Newton updating method. The initial point is the neutral tap position for all SVRs. The objective function portrays the numerical representation of the gross magnitude for the voltage profile. The equality constraints will be the power flow solution and the inequality constraints the voltage limits.

$$
\min \left(\sum_{1}^{i}\left|V_{i, R}\right|+\sum_{1}^{i}\left|V_{i, S}\right|+\sum_{1}^{i}\left|V_{i, T}\right|\right)
$$

subject to:

$$
\begin{aligned}
\mathbf{P}_{a b c}-\operatorname{real}\left(\mathbf { A } \mathbf { V } _ { \alpha \beta 0 } \circ \operatorname { c o n j } \left[\mathbf{A I}_{\left.\left.L_{\alpha \beta 0}\right]\right)}\right.\right. & =0 \\
\mathbf{M z} \mathbf{z}^{T} & =0 \\
\mathbf{Q}_{a b c}-\operatorname{imag}\left(\mathbf{A} \mathbf{V}_{\alpha \beta 0} \circ \operatorname{conj}\left[\mathbf{A} \mathbf{I}_{L_{\alpha \beta 0}}\right]\right) & =0 \\
V_{\text {min }} \leq\left|V_{i, R S T}\right| & \leq V_{\text {max }}
\end{aligned}
$$

All SVR have been configured with $\pm 10 \%$ regulation range , distributed in 32 steps of $\pm 5 / 8 \%$ variation per step ( 0 to 16 for the raise position and -16 to 0 for the lower position), while the admissible voltage variation goes from $\left|V_{\min }\right|=0.95$ to $\left|V_{\max }\right|=1.05$ per unit.

To validate the algorithm a modified version of the IEEE 123 Node Test Feeder (Fig. 2) is selected. Six grounded-wye SVRs have been embedded along the feeder. Most loads in residential distribution are phase-to-neutral, likewise dwellings. For the sake of simplicity, potential existing delta loads, such as motors for building services, are not considered. Anyhow, their inclusion would not have any impact on the optimization, as connection types are not decisive. For a real implementation of this method, the load profiles obtained using GenMIX software [8] should be replaced by measurements obtained from SmartMeters or other advance metering infrastructure devices. A representative sample of 600 different consumption patterns has been simulated. The dwellings are randomly assigned in groups to emulate blocks of apartments and are later distributed between the three phases to achieve diverse unbalance degrees. Explained in [1], the general or hybrid model is basically a combination of the three typical constant load models: constant power (PQ), constant impedance (ZL) and constant current (IL). In terms of current it can be expressed as:

$$
I=\% P Q\left|I_{P Q}\right|+\% Z L\left|I_{Z L}\right|+\% I L\left|I_{I L}\right| \quad \underline{\mid \delta-\varphi}
$$

where $\delta$ is the voltage angle and $\varphi$ the constant load power 


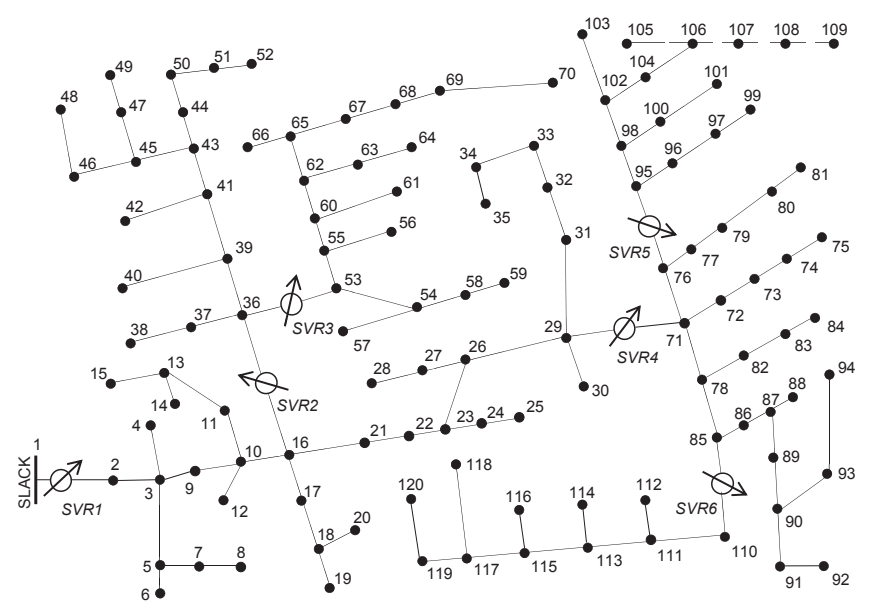

Fig. 2: Modified IEEE 123 Node Test Feeder.

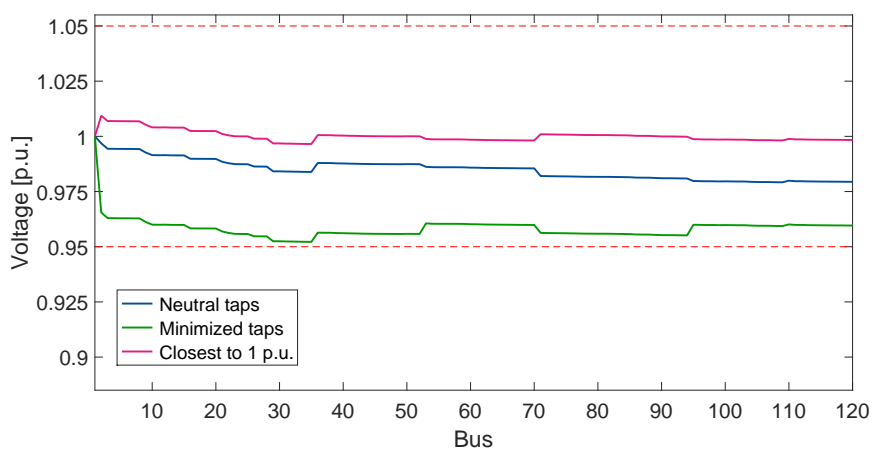

Fig. 3: Voltage profiles comparison under "low loading".

factor. The dwellings distribution consists of $80 \% \mathrm{PQ}$ (power electronics related appliances) and $20 \%$ shared out between the ZL and IL. Since IL devices are rare in common households, the ZL model (lighting/heating) makes up for most of the $20 \%$ for the vast majority of the loads. The following list summarizes the studies carried out during the defined 24-hour span period:

1) Voltage profile analysis.

2) Power injected/consumption/losses comparison.

3) Feeder currents.

4) Degree of unbalance of voltages and currents.

5) Tap configuration.

6) Importance of load location.

An alternative approach has also been taken into account, by minimizing the distance of all voltage magnitudes to $1 \mathrm{pu}$.

\section{RESUlts}

Due to similar load conditions in some hours, two cases are considered: "low loading" (Fig. 3) and "heavy loading" (Fig. 4). Under neutral tap configuration, "heavy load" scenarios translate into bounds violation, while at "low loading" the SVRs would not be mandatory to fulfill the voltage requirements. It can be seen in the plotted phase $\mathrm{R}$ voltage, the minimization success, regardless the load condition. TABLE

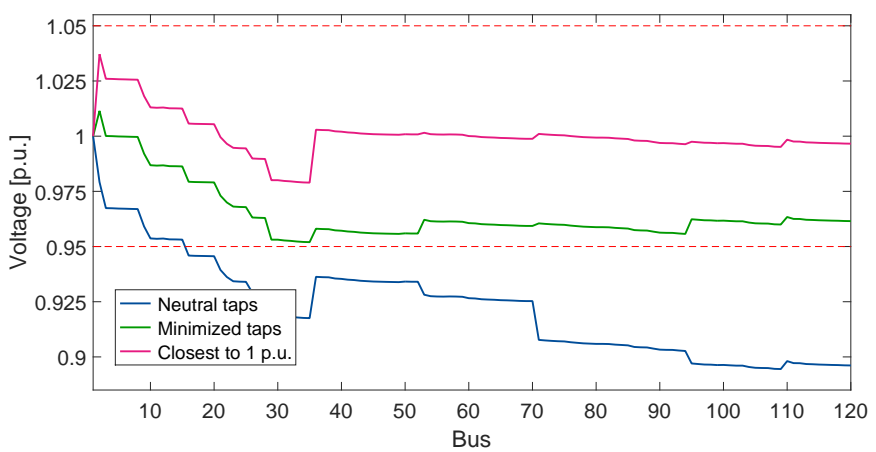

Fig. 4: Voltage profiles comparison under "heavy loading".

I shows power and current at the slack, power consumption and power losses for several hours. At "low loading" (e.g. hours 02,15$)$, the process makes overall power injection/consumption and slack current decreases, while feeder losses slightly increase; The lower the voltage profile at neutral taps, the lower the optimization effects. When the grid is subjected to "heavy loading" conditions (e.g. hours 09, 21), returning the voltage back to the admissible range necessarily increases their demand, due to the general load model nature. The lower the profile at neutral conditions, the greater the power consumption and slack currents after the minimization. The only benefit in these cases would be the reduction of feeder losses. Anyhow, keeping the voltage profile around the 0.95 per unit band guarantees the lowest power consumption. Looking at 24 hours, if the SVRs are disregarded, the feeder demands an hourly average of $2918 \mathrm{~kW}$ with a dwelling consumption of $2811.92 \mathrm{~kW}$, but fails in meeting the voltage constraints at hours $07,08,09,20,21,22$ and 23. Whit optimized profiles, the averages are $2923.67 \mathrm{~kW}$ at the slack and $2818.87 \mathrm{~kW}$ of load demand, without voltage violations. Even though the consumption is greater after the minimization, mainly due to the extremely heavy load conditions at peak hours, the strategy guarantees the least consumption while successfully regulating the voltages, as it was expected. The principal effect of the minimization in terms of unbalance degree results in more constant and delimited profiles, as verified in Fig. 5. Comparing neutral scenarios, all statistical

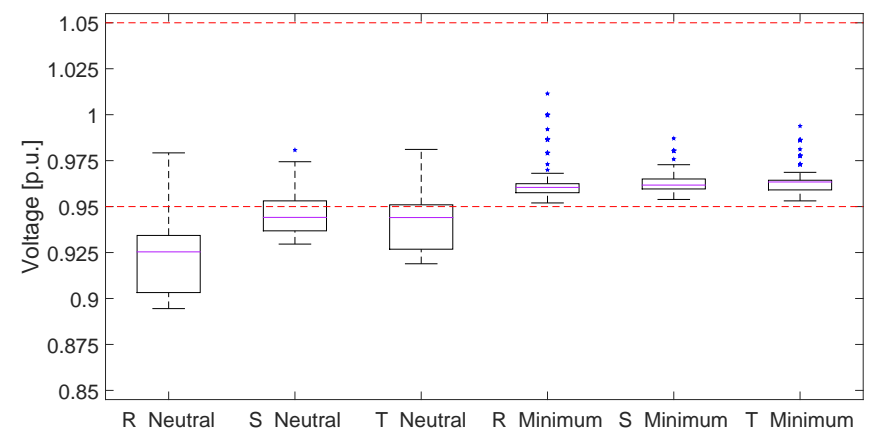

Fig. 5: Statistical analysis of voltage profiles.

variables display a more compact range for the optimized 
TABLE I: Results comparison.

\begin{tabular}{|c|c|c|c|c|c|c|c|c|c|c|}
\hline & & $\begin{array}{c}\sum\left|V_{i}\right| \\
\text { [p.u.] }\end{array}$ & $\begin{array}{c}\left|I_{\text {slack }}\right| \\
{[\mathrm{A}]}\end{array}$ & $\begin{array}{c}\Delta\left|I_{\text {slack }}\right| \\
{[\mathrm{A}]}\end{array}$ & $\begin{array}{c}P_{\text {slack }} \\
{[\mathrm{kW}]}\end{array}$ & $\begin{array}{c}\Delta P_{\text {slack }} \\
{[\mathrm{kW}]}\end{array}$ & $\begin{array}{c}P_{\text {loads }} \\
{[\mathrm{kW}]}\end{array}$ & $\begin{array}{c}\Delta P_{\text {loads }} \\
{[\mathrm{kW}]}\end{array}$ & $\begin{array}{c}P_{\text {losses }} \\
{[\mathrm{kW}]}\end{array}$ & $\begin{array}{c}\Delta P_{\text {losses }} \\
{[\mathrm{kW}]}\end{array}$ \\
\hline \multirow{3}{*}{ Hour 02} & Neutral taps & 118.195 & 6.549 & & 434.460 & & 428.200 & & 6.260 & \\
\hline & Minimized taps & 114.991 & 6.494 & -0.055 & 430.740 & -3.720 & 424.190 & -4.010 & 6.550 & +0.290 \\
\hline & Closest to 1 p.u. & 120.012 & 6.583 & +0.034 & 436.710 & +2.250 & 430.550 & +2.350 & 5.860 & -0.400 \\
\hline \multirow{3}{*}{ Hour 09} & Neutral taps & 110.678 & 27.561 & & 1799.300 & & 1687.000 & & 112.300 & \\
\hline & Minimized taps & 115.770 & 27.817 & +0.256 & 1819.400 & +20.100 & 1712.300 & +25.300 & 107.100 & -5.200 \\
\hline & Closest to 1 p.u. & 120.028 & 28.062 & +0.501 & 1837.700 & +38.400 & 1734.100 & +47.100 & 103.600 & -8.700 \\
\hline \multirow{3}{*}{ Hour 15} & Neutral taps & 117.048 & 10.179 & & 674.300 & & 658.900 & & 15.400 & \\
\hline & Minimized taps & 115.115 & 10.132 & -0.047 & 671.120 & -3.180 & 655.220 & -3.680 & 15.900 & +0.500 \\
\hline & Closest to 1 p.u. & 120.329 & 10.272 & +0.093 & 680.550 & +6.250 & 665.440 & +6.540 & 15.110 & -0.290 \\
\hline \multirow{3}{*}{ Hour 19} & Neutral taps & 114.812 & 17.112 & & 1129.000 & & 1086.700 & & 42.300 & \\
\hline & Minimized taps & 115.462 & 17.146 & +0.034 & 1131.400 & +2.400 & 1088.800 & +2.100 & 42.600 & +0.300 \\
\hline & Closest to 1 p.u. & 120.510 & 17.361 & +0.249 & 1146.200 & +17.200 & 1105.200 & +18.500 & 41.000 & -1.300 \\
\hline \multirow{3}{*}{ Hour 21} & Neutral taps & 106.780 & 36.157 & & 2329.600 & & 2135.400 & & 194.200 & \\
\hline & Minimized taps & 116.511 & 36.549 & +0.392 & 2372.600 & +41.000 & 2196.500 & +61.100 & 176.100 & -18.100 \\
\hline & Closest to 1 p.u. & 119.614 & 36.889 & +0.732 & 2390.800 & +63.200 & 2216.700 & +81.300 & 174.100 & -20.100 \\
\hline
\end{tabular}

profiles. Overall, the results are predominantly flat voltage profiles. The minimization also infers the importance of the location of the SVRs. Looking at the tap positions in TABLE II, the SVR at substation level shows the biggest impact on the degree of unbalance of the voltage profiles of each phase. Tap settings of the other regulators are similar or even the same among phases, and they simply correct the possible voltage deviations at their respective zones. The dwellings location

TABLE II: Minimized tap positions at specified hours.

\begin{tabular}{|c|c|c|c|c|c|c|}
\hline SVR & 1 & 2 & 3 & 4 & 5 & 6 \\
\hline PHASE & R S T & R S T & R S T & R S T & R S T & R S T \\
\hline Hour 02 & $-5-6 \quad 6$ & $\begin{array}{lll}0 & 0 & 0\end{array}$ & 111 & 111 & 111 & 111 \\
\hline Hour 09 & $\begin{array}{lll}5 & 1 & 2\end{array}$ & $\begin{array}{lll}-2 & 0 & -1\end{array}$ & 212 & 222 & 222 & 333 \\
\hline Hour 15 & $-3-5-4$ & $\begin{array}{lll}-1 & 0 & 0\end{array}$ & 111 & 111 & 111 & 111 \\
\hline Hour 19 & $0-3-2$ & $\begin{array}{lll}-1 & 0 & -1\end{array}$ & 111 & 111 & 111 & 222 \\
\hline Hour 21 & 1046 & $-30-2$ & 222 & 232 & 222 & 433 \\
\hline
\end{tabular}

in the feeder has an influence over individual daily voltage profiles and power consumption. For the 24-hour span, Fig. 6 compares phase $\mathrm{R}$ voltage profiles of several nodes of the grid under neutral and minimized tap settings.

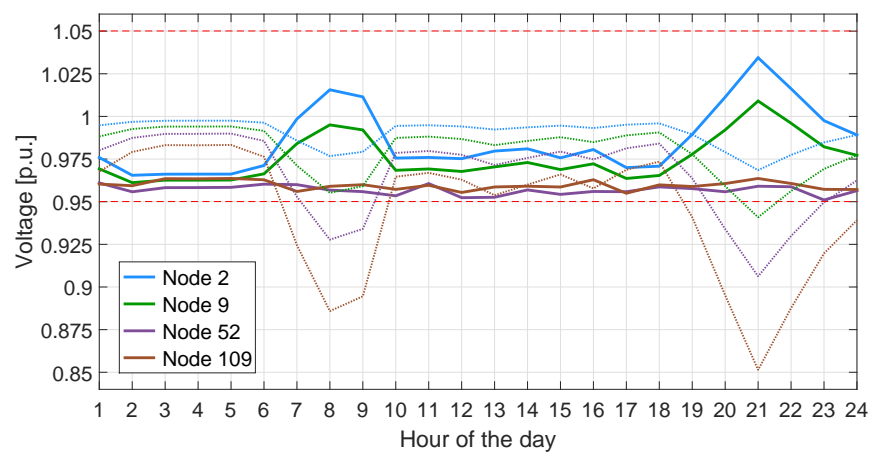

Fig. 6: Daily voltage profile at different nodes.

\section{CONCLUSION}

The authors propose a minimization algorithm that successfully computes the tap positions of SVRs to minimize the voltage profiles of distribution feeders, operating inside the stipulated regulation band as well as trying to adjust the closest to the lower limit. By evaluating the algorithm on a large scale grid, several operating aspects were analyzed. The use of the general load model and the daily load demand prediction contributed to achieve more realistic results than classic constant PQ load studies. It is concluded that a critical factor in determining to what extent the minimized voltage profiles would influence feeder behavior is the load conditions. Specifically, for residential distribution feeders, both appropriate demand prediction and load modeling are mandatory if reallife situations are to be resembled. A centralized control lowest power injections/consumptions improving the phase voltage balancing. Dwelling location becomes the most controversial aspect, as both voltage profiles and power demand depend on the distance between the main substation regulator and the dwelling.

\section{REFERENCES}

[1] W. H. Kersting, Distribution System Modeling and Analysis. CRC Press, 2001.

[2] B. A. Robbins, H. Zhu, and A. D. Domínguez-García, "Optimal tap setting of voltage regulation transformers in unbalanced distribution systems," Power Systems, IEEE Transactions on, vol. 31, no. 1, pp. 256267, Jan. 2016

[3] C. A. N. Pereira and C. A. Castro, "Optimal placement of voltage regulators in distribution systems," IEEE Bucharest Power Tech Conference, 2009.

[4] R. Shigenobu and T. Funabashi, "Multi-objective optimization of SVR considering optimum placement and weather conditions in distribution systems," Intelligent System Application to Power Systems (ISAP), 18th International Conference on, Sept. 2015.

[5] M. I. Hossain, R. Yan, and T. K. Saha, "Investigation of the interaction between step voltage regulators and large-scale photovoltaic systems regarding voltage regulation and unbalance," IET Renewable Power Generation, vol. 10, no. 3, pp. 299-309, Mar. 2016.

[6] P. Arboleya, C. Gonzalez-Moran, and M. Coto, "Modeling facts for power flow purposes: A common framework," International Journal of Electrical Power and Energy Systems, vol. 63, pp. 293 - 301, 2014.

[7] — , "Unbalanced power flow in distribution systems with embedded transformers using the complex theory in $\alpha \beta 0$ stationary reference frame," IEEE Trans. Power Syst., vol. PP, no. 99, pp. 1-11, may 2014. 